\title{
Predicting factors of intradialytic hypotension in the last half of hemodialysis therapy
}

\author{
Yumi Aoyama ${ }^{1,2}$, Katsuya Kajimoto ${ }^{3}$, Maiko Hori ${ }^{1,4}$, Shigeru Otsubo ${ }^{5,6^{*}}$ and Kosaku Nitta ${ }^{1}$
}

\begin{abstract}
Background: Intradialytic hypotension (IDH) is one of the most frequent complications of hemodialysis. IDH alters a patient's quality of life and has a strong association with mortality. IDH mainly occurs when ultrafiltration during hemodialysis exceeds the pace of the plasma refill. Ultrasonography has been used to evaluate the intravascular volume status. We evaluated the association between IDH and baseline laboratory data or ultrasound findings during IDH during the latter half of a hemodialysis session.

Methods: Sixty-two patients who underwent hemodialysis were enrolled in this study. The peak early mitral inflow wave velocity (peak E-velocity), the inferior vena cava (IVC) dimensions, and number of B-lines were estimated at two time points (just after the start and at the midpoint of the hemodialysis session). We compared the parameters between the patients without IDH throughout the hemodialysis session (non-IDH group) and patients with IDH only during the latter half of the hemodialysis session (IDH in the latter half group).

Results: Of the 62 study participants, 28 patients were in non-IDH group, and 16 patients were in IDH in the latter half group. The serum sodium level was significantly lower in the IDH in the latter half group (135 $\pm 4 \mathrm{mEg} / \mathrm{L}$ ) than in the non-IDH group $(138 \pm 4 \mathrm{mEq} / \mathrm{L})(P=0.015)$. The rate of decrease of the peak E-velocity was significantly higher in the IDH in the latter half group $(0.33 \pm 0.13)$ than in the non-IDH group $(0.24 \pm 0.14)(P=0.037)$. The best cutoff point of the serum level of sodium was $137 \mathrm{mEq} / \mathrm{L}$, with a sensitivity of $68.8 \%$ and specificity of $64.3 \%$. The area under the receiver operating characteristic (ROC) curve was 0.71 . The best cutoff point of the rate of decrease of the peak E-velocity was 0.295 , with a sensitivity of $73.3 \%$ and a specificity of $71.4 \%$. The area under the ROC curve was 0.71 .
\end{abstract}

Conclusions: A low serum level of sodium at baseline and an early decrease in the peak E-velocity during hemodialysis may be related to intradialytic hypotension in the latter half of a hemodialysis session. Careful observation may be needed in the hemodialysis patients in whom the serum level of sodium is below $137 \mathrm{mEq} / \mathrm{L}$ or the rate of decrease of the peak E-velocity is over 0.295.

Keywords: Lung echo, Intradialytic hypotension, Peak E-velocity, B-line, Hemodialysis, Hyponatremia

\footnotetext{
* Correspondence: sotsubo33@gmail.com

${ }^{5}$ Department of Blood Purification, Tohto Sangenjaya Clinic, 2-13-2 Taishido,

Setagaya-ku, Tokyo 154-0004, Japan

${ }^{6}$ Department of Clinical Engineering, Faculty of Human Care at Makuhari,

Tohto University, Chiba, Japan

Full list of author information is available at the end of the article
}

C C The Author(s). 2021 Open Access This article is licensed under a Creative Commons Attribution 4.0 International License, which permits use, sharing, adaptation, distribution and reproduction in any medium or format, as long as you give appropriate credit to the original author(s) and the source, provide a link to the Creative Commons licence, and indicate if changes were made. The images or other third party material in this article are included in the article's Creative Commons licence, unless indicated otherwise in a credit line to the material. If material is not included in the article's Creative Commons licence and your intended use is not permitted by statutory regulation or exceeds the permitted use, you will need to obtain permission directly from the copyright holder. To view a copy of this licence, visit http://creativecommons.org/licenses/by/4.0/. The Creative Commons Public Domain Dedication waiver (http://creativecommons.org/publicdomain/zero/1.0/) applies to the data made available in this article, unless otherwise stated in a credit line to the data. 


\section{Background}

Intradialytic hypotension (IDH) is one of the most frequent complications of hemodialysis and has been estimated to occur in $5-32.5 \%$ of hemodialysis sessions [14]. IDH alters a patient's quality of life; it is known to induce ischemic brain injury and myocardial stunning in hemodialysis patients [3-6], thereby showing a strong association with mortality [2, 3, 7]. IDH mainly occurs under the following conditions: when ultrafiltration during hemodialysis exceeds the pace of the plasma refill; failure of physiologic compensatory mechanisms, including autonomic/baroreceptor failure; cardiac dysfunction; impaired vasoconstriction; reduced effective circulating volume. Ultrasonography has been used to evaluate the intravascular volume status. The inferior vena cava (IVC) dimensions represent the right ventricular preload [8], while the peak early mitral inflow wave velocity (peak E-velocity) represents the left ventricular preload $[9,10]$. In addition, lung ultrasound has recently been shown to be a useful, noninvasive technique for assessing extravascular lung water [11]. The most commonly observed finding is a comet tail artifact fanning out from the lung-wall interface and spreading upwards towards the edge of the screen, named a "B-line" [12]. The number of B-lines observed in lung ultrasound represents the severity of pulmonary congestion $[13,14]$. In this study, echographic examinations were estimated at 2 time points during a hemodialysis session (at the start and at a time point half way through the dialysis session [the midpoint]). We evaluated the association between IDH and baseline laboratory data or ultrasound findings during IDH during the latter half of a hemodialysis session.

\section{Methods}

We performed a retrospective, observational cohort study to investigate the factors of IDH in the last half of hemodialysis therapy. Sixty-two patients who underwent hemodialysis at Sekikawa Hospital and whose dialysis data were available were enrolled in this study. Clinical data were collected from the patients' clinical records. Hypertension was defined by a systolic blood pressure of $140 \mathrm{~mm} \mathrm{Hg}$ or higher, diastolic blood pressure of 90 $\mathrm{mm} \mathrm{Hg}$ or higher, and/or current use by the patient of antihypertensive drugs. Diabetes mellitus was defined by a fasting blood glucose level of $\geq 126 \mathrm{mg} / \mathrm{dL}$, non-fasting blood glucose level of $\geq 200 \mathrm{mg} / \mathrm{dL}$, and/or current use by the patient of antidiabetic medication. Dyslipidemia was defined by a serum low-density lipoprotein cholesterol level of $\geq 140 \mathrm{mg} / \mathrm{dL}$, high-density lipoprotein cholesterol level of $<40 \mathrm{mg} / \mathrm{dL}$, triglyceride level of $\geq 150 \mathrm{mg} /$ $\mathrm{dL}$, and/or current use by the patient of lipid-modifying medication. IDH was defined according to the EBPG guideline, which was same as the JSDT guideline, by a decrease of the systolic blood pressure by $>20 \mathrm{~mm} \mathrm{Hg}$ or decrease of the mean arterial pressure by $>10 \mathrm{~mm}$ $\mathrm{Hg}$, associated with a clinical event and/or the need for nursing intervention [15].

A peripheral blood sample was obtained from each patient before the start of the first hemodialysis session of the week. A lung ultrasound examination was performed on the same day as the peripheral blood sample collection. Bilateral scanning of the anterior and lateral chest walls was performed with the patient in the supine position. An intercostal scan with maximum extension of the visual pleural line was performed. The chest wall was divided into 8 areas ( 2 anterior and 2 lateral areas per side), and each of these areas was scanned [16]. The number of B-lines was counted as described in a previous report [17-19]. A cardiologist, who is blind to the result of blood peripheral blood sample, performed echocardiography test. The peak E-velocity, IVC dimensions, and number of B-lines were estimated at two time points (just after the start and at the midpoint of the hemodialysis session). Each of the rates of decrease from the start to the midpoint of the dialysis session was also evaluated. The other echocardiographic measurements were obtained at the start of the hemodialysis session.

All the study participants were divided to 3 groups according to their blood pressure status during the hemodialysis session. Patients without IDH throughout the hemodialysis session (non-IDH group), patients with IDH only during the latter half of the hemodialysis session (IDH in the latter half group), and patients with IDH during the first half of the dialysis session. We compared the parameters between the non-IDH group and the IDH in the latter half group.

Data are expressed as the means \pm standard deviation or medians (interquartile ranges). The statistical significances of differences were determined using a two-sided paired $t$-test. For non-normally distributed variables, a two-sided Wilcoxon's signed rank test was used. The chi-square or Fisher's exact probability test was used for categorical data. A receiver operating characteristic (ROC) analysis was performed to assess the cutoff point for IDH in the latter half of the hemodialysis session, and the area under the curve, sensitivity, and specificity were estimated. All the statistical calculations were performed using the JMP 5.1 software. $P$ values of less than 0.05 were considered as being indicative of statistical significance. This study was conducted in accordance with the principles of the Declaration of Helsinki and with the approval of the research ethics committee of Sekikawa Hospital (Approved No. H2705).

\section{Results}

Of the 62 study participants, while no IDH was observed at any time point during the dialysis in 28 patients (nonIDH group), 16 patients developed IDH only during the 
latter half of the hemodialysis session (IDH in the latter half group). The remaining 18 developed IDH during the first half of the dialysis session. Medical interventions for IDH in the latter half group were the following: reduction of dialysate temperature in 5 cases, elevation of the lower limbs in 4 cases, reduction of filtration flow rate in 3 cases, infusions of fluid in 2 cases, and administration of a vasopressor agent in 2 cases.

Comparison of the background characteristics between the two groups are shown in Table 1. The gender distribution, mean age, and mean dialysis vintage were not statistically significantly different. The proportion of patients with diabetes mellitus tended to be higher in the IDH in the latter half group (62.5\%) than in the nonIDH group (39.3\%), although the difference was not statistically significant $(P=0.138)$. The percentages of patients with hypertension, dyslipidemia, and ischemic heart disease were not significantly different between the two groups. The amount of removed water/treatment time and amount of removed water/treatment time/body weight during the first half of the dialysis session were not statistically significantly different between the two groups.

A comparison of the laboratory data is shown in Table 2. There was no significant difference in the hemoglobin concentration or serum level of albumin, urea nitrogen, creatinine, or C-reactive protein between the two groups. However, the serum sodium level was significantly lower in the IDH in the latter half group (135 \pm 4 $\mathrm{mEq} / \mathrm{L})$ than in the non-IDH group $(138 \pm 4 \mathrm{mEq} / \mathrm{L})(P$

Table 1 Comparison of the background characteristics

\begin{tabular}{|c|c|c|c|}
\hline Characteristic & IDH in the latter half & Non-IDH & $P$ value \\
\hline Number & 16 & 28 & NS \\
\hline Gender (M/F) & $8 / 8$ & $17 / 11$ & NS \\
\hline Age (year) & $74.7 \pm 11.3$ & $76.4 \pm 10.0$ & NS \\
\hline Dialysis vintage (year) & $4.3[0.5-8.2]$ & $3.1[2.4-9.3]$ & NS \\
\hline Hypertension, $n(\%)$ & $13(81.3)$ & $24(85.7)$ & NS \\
\hline Diabetes mellitus, $n(\%)$ & $10(62.5)$ & $11(39.3)$ & NS \\
\hline Dyslipidemia, n (\%) & $3(18.8)$ & $4(14.3)$ & NS \\
\hline Ischemic heart disease, $n(\%)$ & $3(18.8)$ & $6(21.4)$ & NS \\
\hline \multicolumn{4}{|l|}{ Use of anti-hypertensive drug, $n(\%)$} \\
\hline All types & $12(75.0)$ & $24(85.6)$ & NS \\
\hline RAS inhibitor & $9(56.3)$ & $9(32.1)$ & NS \\
\hline \multicolumn{4}{|l|}{ History of coronary artery disease, $n(\%)$} \\
\hline $\mathrm{PCl}$ & $2(12.5)$ & $2(7.1)$ & NS \\
\hline CABG & $2(12.5)$ & $0(0)$ & NS \\
\hline Body weight $(\mathrm{kg})^{\mathrm{a}}$ & $51.6 \pm 8.4$ & $51.9 \pm 10.1$ & NS \\
\hline Systolic blood pressure $(\mathrm{mmHg})^{a}$ & $153 \pm 29$ & $137 \pm 23$ & NS \\
\hline Diastolic blood pressure $(\mathrm{mmHg})^{\mathrm{a}}$ & $75 \pm 16$ & $70 \pm 16$ & NS \\
\hline Heart rate (beat/minute) ${ }^{a}$ & $77 \pm 10$ & $75 \pm 15$ & NS \\
\hline Dialysis time & $3.8 \pm 0.5$ & $3.8 \pm 0.4$ & NS \\
\hline Amount of removed water/treatment time $(\mathrm{kg} / \mathrm{h})^{\mathrm{b}}$ & $0.60 \pm 0.21$ & $0.50 \pm 0.26$ & NS \\
\hline Amount of removed water/treatment time/BW $(1 / h)^{b}$ & $0.012 \pm 0.003$ & $0.010 \pm 0.004$ & NS \\
\hline
\end{tabular}

Mean \pm SD, median [interquartile range]

$\mathrm{PCl}$ percutaneous coronary intervention, $C A B G$ coronary artery bypass grafting, $B W$ body weight

${ }^{a}$ At the start of dialysis therapy

${ }^{b}$ During first half of dialysis session 
Table 2 Comparison of blood sample

\begin{tabular}{llll}
\hline Characteristic & IDH in the latter half & Non-IDH & $P$ value \\
\hline Albumin $(\mathrm{g} / \mathrm{dL})$ & $3.2 \pm 0.4$ & $3.0 \pm 0.6$ & $\mathrm{NS}$ \\
Urea nitrogen $(\mathrm{mg} / \mathrm{dL})$ & $56.3 \pm 23.6$ & $49.7 \pm 16.3$ & $\mathrm{NS}$ \\
Creatinine $(\mathrm{mg} / \mathrm{dL})$ & $7.5 \pm 2.8$ & $7.4 \pm 2.5$ & $\mathrm{NS}$ \\
Sodium $(\mathrm{mEg} / \mathrm{L})$ & $135 \pm 4$ & $138 \pm 4$ & 0.015 \\
C-reactive protein $(\mathrm{mg} / \mathrm{dL})$ & $0.29[0.09-1.27]$ & $0.29[0.11-0.52]$ & $\mathrm{NS}$ \\
Hemoglobin $(\mathrm{g} / \mathrm{dL})$ & $10.7 \pm 1.2$ & $10.3 \pm 1.0$ & $\mathrm{NS}$ \\
\hline
\end{tabular}

Mean $\pm S D$, median [interquartile range]

$=0.015)$. The serum albumin was low in this study participants. The serum level of albumin was $3.0 \pm 0.6 \mathrm{~g} / \mathrm{dL}$ in non-diabetes mellitus patients $(n=33)$ and $3.1 \pm 0.5 \mathrm{~g} /$ $\mathrm{dL}$ in diabetes mellitus patients $(n=29)$. There was no statistical difference between the two groups.

A comparison of the echocardiographic indices between the two groups is shown in Table 3. At the start of hemodialysis therapy, the left ventricular ejection fraction was $57.4 \pm 15.2$ in the IDH in the latter half group and $61.0 \pm 14.6$ in the non-IDH group, with no statistically significant difference between the two groups. The peak E-velocity, IVC dimensions, and number of B-lines at the start of the dialysis session were also not significantly different between the two groups. At the midpoint of the hemodialysis session, the peak E-velocity tended to be lower in the IDH in the latter half group $(52 \pm 24$ $\mathrm{cm} / \mathrm{s})$ than in the non-IDH group $(60 \pm 17 \mathrm{~cm} / \mathrm{s})$, although the difference was not statistically significant $(P$ $=0.213$ ). The IVC dimensions at the midpoint of the hemodialysis session also tended to be lower in the IDH in the latter half group $(11 \pm 2 \mathrm{~mm})$ than in the nonIDH group $(13 \pm 4 \mathrm{~mm})$, although this difference was also not statistically significant $(P=0.249)$. The number of B-lines at the midpoint of the hemodialysis session was not significantly different between the two groups. The rate of decrease of the peak E-velocity was significantly higher in the IDH in the latter half group $(0.33 \pm$ $0.13)$ than in the non-IDH group $(0.24 \pm 0.14)(P=$ 0.037). The rate of decrease of the IVC dimensions tended to be higher in the IDH in the latter half group $(0.25 \pm 0.14)$ than in the non-IDH group $(0.18 \pm 0.17)$, but this difference was also not statistically significant $(P$

Table 3 Comparison of echocardiographic indices

\begin{tabular}{|c|c|c|c|}
\hline & IDH in the latter half & Non-IDH & $P$ value \\
\hline \multicolumn{4}{|l|}{ At the start } \\
\hline LVDd (mm) & $46.1 \pm 5.4$ & $46.3 \pm 5.3$ & NS \\
\hline LVDs (mm) & $31.3 \pm 6.7$ & $31.2 \pm 7.3$ & NS \\
\hline LVEF (\%) & $57.4 \pm 15.2$ & $61.0 \pm 14.6$ & NS \\
\hline Maximum WT (mm) & $11.9 \pm 1.4$ & $11.1 \pm 1.6$ & NS \\
\hline Peak E-velocity (cm/s) & $77 \pm 30$ & $81 \pm 23$ & NS \\
\hline IVC-ex (mm) & $16 \pm 3$ & $15 \pm 4$ & NS \\
\hline B-lines & $13 \pm 5$ & $11 \pm 4$ & NS \\
\hline \multicolumn{4}{|l|}{ At the middle } \\
\hline Peak E-velocity (cm/sec) & $52 \pm 24$ & $60 \pm 17$ & NS \\
\hline IVC-ex dimension (mm) & $11 \pm 2$ & $13 \pm 4$ & NS \\
\hline B-lines & $10 \pm 4$ & $9 \pm 3$ & NS \\
\hline \multicolumn{4}{|c|}{ Decreasing rate (start to the midpoint) } \\
\hline Peak E-velocity & $0.33 \pm 0.13$ & $0.24 \pm 0.14$ & 0.037 \\
\hline IVC-ex dimension & $0.25 \pm 0.14$ & $0.18 \pm 0.17$ & NS \\
\hline B-lines & $0.20 \pm 0.19$ & $0.19 \pm 0.20$ & NS \\
\hline
\end{tabular}

Mean \pm SD

Decreasing rate (start to the midpoint) $=$ (at the start-at the middle)/at the start

LVDd left ventricular end-diastolic dimension, LVDs left ventricular end-systolic dimension, LVEF left ventricular ejection fraction, WT wall thickness, IVC inferior vena cava dimensions, ex exhalation 
$=0.135)$. There was also no significant difference in the rate of decrease of the number of B-lines between the two groups.

Figure 1 illustrates the ROC analysis performed to determine the optimum cutoff points for the diagnosis of IDH in the latter half of the hemodialysis session. The best cutoff point of the serum level of sodium was 137 $\mathrm{mEq} / \mathrm{L}$, with a sensitivity of $68.8 \%$ and specificity of $64.3 \%$. The area under the ROC curve was 0.71 . The best cutoff point of the rate of decrease of the peak E-velocity was 0.295 , with a sensitivity of $73.3 \%$ and specificity of $71.4 \%$. The area under the ROC curve was 0.71 .

\section{Discussion}

In this study, we demonstrated that the serum level of sodium and rate of decrease of the peak E-velocity were higher in the IDH in the latter half group than in the non-IDH group. The cutoff value for predicting IDH in the latter half of the hemodialysis session was $137 \mathrm{mEq} /$ $\mathrm{L}$ for the serum level of sodium and 0.295 for the rate of decrease of the peak E velocity. The likelihood of diabetes mellitus tended to be higher in the IDH in the latter half group than in the non-IDH group.

IDH is caused by a decrease of the cardiac output or decrease of the peripheral vascular resistance. Cardiac output decreases as the intravascular volume decreases. The change in the intravascular volume during hemodialysis is determined by the balance among the ultrafiltration rate, decrease in plasma osmolality, and rate of plasma refilling from the interstitium. Both the IVC dimensions and the peak E-velocity are related to the intravascular volume; the IVC dimensions represent the right ventricular preload [8], whereas the peak E- velocity represents the left ventricular preload $[9,10]$. At the midpoint of the hemodialysis session, the peak Evelocity and IVC dimensions tend to be lower in the IDH in the latter half group than in the non-IDH group. The differences might have been statistically significant if the number of study participants were higher. The rate of decrease of the peak E-velocity was higher in the IDH in the latter half group than in the non-IDH group. The rate of change of this parameter may be more useful than the absolute value for predicting the risk of IDH. No significant difference in the rate of decrease of the IVC dimensions was observed between the two groups. Both the peak E-velocity and IVC dimensions reflect the intravascular volume, but the peak E-velocity seems to be a more sensitive indicator of the intravascular volume than the IVC dimensions. On the other hand, B-lines represent extravascular lung water. We previously reported that the IVC dimensions and peak E-velocity decrease mainly during the first half of the dialysis session, while significant decrease in the number of B-lines, which reflects lung congestion, is observed mainly during the latter half of the dialysis session [18]. This finding may be largely dependent on the time lag in plasma refilling. In this study, there was no significant difference in the rate of decrease of the B-lines between the two groups. This may imply that in the IDH in the latter half group, the plasma refilling volume was not sufficient to maintain an adequate intravascular volume during the last half of the dialysis session. Actually, the serum level of sodium, which is an important determinant of the plasma osmotic pressure, was significantly lower in the IDH in the latter half group than in the non-IDH group. The lower level of serum sodium may prevent sufficient

\section{a)}

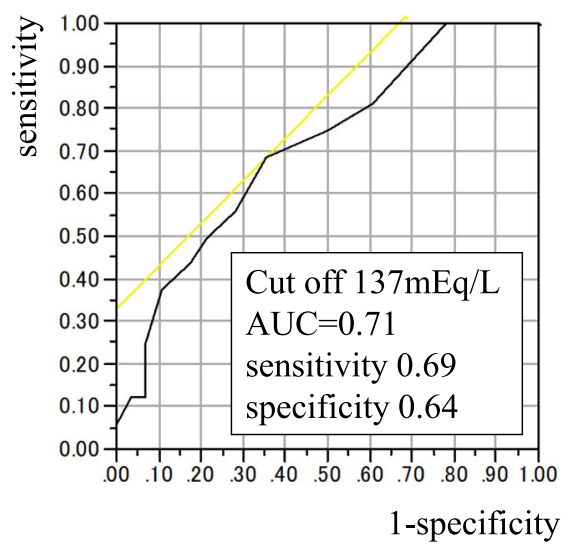

b)

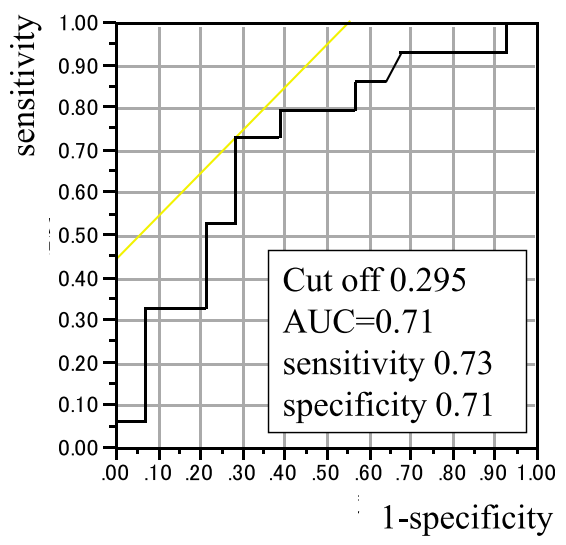

Fig. 1 Receiver operating characteristic (ROC) curve analysis to identify the cutoff points of the serum level of sodium and rate of decrease (start to the midpoint) of the peak E-velocity as predictors of IDH in the latter half of a hemodialysis session. a The ROC curve analysis revealed a cutoff value of $137 \mathrm{mEq} / \mathrm{L}$ and area under the curve of 0.79 for the serum sodium level, with a sensitivity of $69 \%$ and specificity of $64 \%$ for predicting IDH. b The ROC analysis revealed a cutoff value of 0.295 and area under the curve of 0.71 for the rate of decrease of the peak E-velocity, with a sensitivity of $73 \%$ and a specificity of $71 \%$ for predicting IDH. IHD, intradialytic hypotension; AUC, area under the curve 
plasma refilling, which may result in IDH in the latter half of the hemodialysis session. The cutoff point of the serum level of sodium for predicting IDH was $137 \mathrm{mEq} /$ L. Thus, patients with serum sodium levels of below 137 $\mathrm{mEq} / \mathrm{L}$ may require careful observation. In the same way, IDH patients have also previously been reported to show lower serum albumin levels [20]. However, in our study, the serum level of albumin was not significantly different between the IDH in the latter half group and the non-IDH group. The serum level of albumin may be a predictor of IDH in the first half of the hemodialysis session.

Reduced cardiac output also reflected by a reduced left ventricular ejection fraction may be caused by ischemic heart disease, left ventricular hypertrophy, and valvular heart disease, which can thereby represent risk factors for IDH [21, 22]. In this study, the proportion of patients with ischemic heart disease, the mean left ventricular ejection fraction, and the mean value of the maximum wall thickness did not differ between the two groups. It is possible that these are predictors of IDH in the first half of a hemodialysis session.

Decreased peripheral vascular resistance is another cause of IDH. Allergy or biocompatibility of anticoagulants, dialysis membranes, and acetic acid-containing dialysate could decrease the peripheral vascular resistance and cause a drop of the blood pressure just after the start of a hemodialysis session. The normal compensatory increase of the peripheral vascular resistance that occurs to maintain the blood pressure when the intravascular volume falls with a high rate of ultrafiltration may fail to occur due to impaired diabetic autonomic neuropathy in patients with diabetes mellitus. Indeed, diabetes mellitus has been reported as a risk factor for IDH $[3,23]$. In our study, the proportion of patients with diabetes mellitus tended to be higher in the IDH in the latter half group than in the non-IDH group. The differences might have been statistically significant if there were a higher number of study participants.

Our study had some limitations. The sample size was relatively small, and the study was performed at a single institution. To conclude the predicting factors of IDH, other factors such as plasma refilling rate, arrythmias, valvular heart disease, or ejection fraction should be considered. More patients should be addressed using multivariate analysis. The study participants were relatively old, their hemoglobin and serum albumin levels were relatively low, and their serum NT-proBNP levels were relatively high because of the specificity of our institution. We could not estimate exact dry weight. The golden standard method to determine dry weight does not exist, but there are some markers of intravascular volume such as cardiothoracic ratio or serum level of atrial natriuretic peptide. The bioimpedance spectroscopy device enables to derive the extracellular water and intracellular water. But we did not have such data at the same time; setting of dry weight may not be correct in some study participants. We should also divide patients according to their cardiac functions, but we could not because of small study participants. Another limitation of this study was that the investigator who performed lung ultrasound was not blind for clinical information of the patients such as age, gender, or clinical diagnosis. In addition, the limitations of lung ultrasound are essentially patient-dependent. Obese patients may be more difficult to examine because of the thickness of their ribcages and soft tissues. As we focused on IDH in latter half of a hemodialysis session, we could not assess the predictors of IDH occurring just after the start of a hemodialysis session.

\section{Conclusions}

A low serum level of sodium at baseline and an early decrease in the peak E-velocity during hemodialysis may be related to intradialytic hypotension in the latter half of a hemodialysis session. Careful observation may be needed in the hemodialysis patients in whom the serum level of sodium is below $137 \mathrm{mEq} / \mathrm{L}$ or the rate of decrease of the peak E-velocity is over 0.295.

\section{Abbreviations \\ IHD: Intradialytic hypotension; IVC: Inferior vena cava; Peak E-velocity: Peak early mitral inflow wave velocity; ROC: Receiver operating characteristic}

\section{Acknowledgements}

The authors are very grateful to dialysis staff who understood the clinical importance of this study and who provided high-quality data in Sekikawa Hospital.

\section{Authors' contributions}

YA and SO planned the study, searched the literature, assessed studies, extracted data, analyzed data, and prepared article. KK planned the study, assessed studies, performed echocardiographic examinations, extracted data, and assisted in article preparation. $\mathrm{KN}$ and $\mathrm{MH}$ assisted in article preparation. All authors read and approved the final manuscript.

Funding

This study was not supported by any grants or funding.

\section{Availability of data and materials}

The datasets during and/or analyzed during the current study are available from the corresponding author on reasonable request.

\section{Declarations}

Ethics approval and consent to participate

This study was approved by the research ethics committee of Sekikawa Hospital (Approved No. H2705) and was conducted under the Declaration of Helsinki.

Consent for publication

Not applicable.

Competing interests

The authors declared that they have no competing interest. 


\section{Author details}

'Department of Nephrology, Tokyo Women's Medical University, Tokyo, Japan. ${ }^{2}$ Department of Nephrology, Sangenjaya Hospital, Tokyo, Japan. ${ }^{3}$ Department of Cardiology, Sekikawa Hospital, Tokyo, Japan. ${ }^{4}$ Department of Nephrology, Sekikawa Hospital, Tokyo, Japan. ${ }^{5}$ Department of Blood Purification, Tohto Sangenjaya Clinic, 2-13-2 Taishido, Setagaya-ku, Tokyo 154-0004, Japan. ${ }^{6}$ Department of Clinical Engineering, Faculty of Human Care at Makuhari, Tohto University, Chiba, Japan.

Received: 1 November 2020 Accepted: 29 March 2021

Published online: 09 April 2021

\section{References}

1. Halle MP, Hilaire D, Francois KF, Denis T, Hermine F, Gloria AE. Intradialytic hypotension and associated factors among patients on maintenance hemodialysis: a single-center study in Cameroon. Saudi J Kidney Dis Transpl. 2020;31(1):215-23.

2. Flythe JE, Xue H, Lynch KE, Curhan GC, Brunelli SM. Association of mortality risk with various definitions of intradialytic hypotension. J Am Soc Nephrol. 2015;26:724-34.

3. Sands JJ, Usvyat LA, Sullivan T, et al. Intradialytic hypotension: frequency, sources of variation and correlation with clinical outcome. Hemodial Int. 2014; 18:415-22.

4. Stefánsson BV, Brunelli SM, Cabrera C, et al. Intradialytic hypotension and risk of cardiovascular disease. Clin J Am Soc Nephrol. 2014;9:2124-32.

5. Mizumasa T, Hirakata H, Yoshimitsu T, et al. Dialysis-related hypotension as a cause of progressive frontal lobe atrophy in chronic hemodialysis patients: a 3-year prospective study. Nephron Clin Pract. 2004;97:c23-30.

6. McIntyre CW, Goldsmith DJ. Ischemic brain injury in hemodialysis patients: which is more dangerous, hypertension or intradialytic hypotension? Kidney Int. 2015:87:1109-15.

7. Chou JA, Kalantar-Zadeh K, Mathew AT. A brief review of intradialytic hypotension with a focus on survival. Semin Dial. 2017;30:473-80.

8. Simonson JS, Schiller NB. Sonospirometry: a new method for noninvasive estimation of mean right atrial pressure based on two-dimensional echographic measurements of the inferior vena cava during measured inspiration. J Am Coll Cardiol. 1988;11:557-64.

9. Triulzi MO, Castini D, Ornaghi M, Vitolo E. Effects of preload reduction on mitral flow velocity pattern in normal subjects. Am J Cardiol. 1990;66:9951001.

10. Sztajzel J, Ruedin P, Monin C, Stoermann C, Leski M, Rutishauser W, et al. Effect of altered loading conditions during haemodialysis on left ventricular filling pattern. Eur Heart J. 1993;14:655-61.

11. Alexiadis G, Panagoutsos S, Roumeliotis S, Stibiris I, Markos A, Kantartzi K, et al. Comparison of multiple fluid status assessment methods in patients on chronic hemodialysis. Int Urol Nephrol. 2017:49:525-32.

12. Lichtenstein D. Pneumothorax and introduction to ultrasound signs in the lung. In: Heilmann U, Wilbertz H, Gosling A, editors. General ultrasound in the critically ill. 1st ed. Heidelberg: Springer-Verlag; 2005. p. 105-15.

13. Picano E, Pellikka PA. Ultrasound of extravascular lung water: a new standard for pulmonary congestion. Eur Heart J. 2016;37:2097-104.

14. Kajimoto K, Madeen K, Nakayama T, Tsudo H, Kuroda T, Abe T. Rapid evaluation by lung-cardiac-inferior vena cava $(\mathrm{LCl})$ integrated ultrasound for differentiating heart failure from pulmonary disease as the cause of acute dyspnea in the emergency setting. Cardiovasc Ultrasound. 2012;10:49-51.

15. Kooman J, Basci A, Pizzarelli F, et al. EBPG guideline on haemodynamic instability. Nephrol Dial Transplant. 2007;22(Suppl 2):ii22-44.

16. Volpicelli G, Elbarbary M, Blaivas M, Lichtenstein DA, Mathis G, Kirkpatrick AW, et al. International evidence-based recommendations for point-of-care lung ultrasound. Intensive Care Med. 2012;38:577-91.

17. Kuzuhara S, Otsubo S, Kajimoto K, Akiba T, Nitta K. Association between Blines detected during lung ultrasound and various factors in hemodialysis patients. Ren Replace Ther. 2017;3:17.

18. Kimura K, Kajimoto K, Otsubo S, Akiba T, Nitta K. Associations between fluid removal and number of B-lines, peak early mitral inflow wave velocity, and inferior vena cava dimensions in hemodialysis patients. Ren Replace Ther. 2017;3:54.

19. Kawachi K, Kajimoto K, Otsubo S, Nitta K. Associations between pulmonary congestion on chest ultrasound and survival in hemodialysis patients. Ren Replace Ther. 2019:5:27.
20. Fortin PM, Bassett K, Musini VM. Human albumin for intradialytic hypotension in haemodialysis patients. Cochrane Database Syst Rev. 2010; (11). https://doi.org/10.1002/14651858.CD006758.pub2.

21. Assimon MM, Flythe JE. Intradialytic blood pressure abnormalities: the highs, the lows and all that lies between. Am J Nephrol. 2015;42:337-50.

22. Barberato SH, Misocami M, Pecoits-Filho R. Association between left atrium enlargement and intradialytic hypotension: role of diastolic dysfunction in the hemodynamic complications during hemodialysis. Echocardiography. 2009:26:767-71.

23. van der Sande FM, Dekker MJ, Leunissen KML, Kooman JP. Novel insights into the pathogenesis and prevention of intradialytic hypotension. Blood Purif. 2018;45:230-5.

\section{Publisher's Note}

Springer Nature remains neutral with regard to jurisdictional claims in published maps and institutional affiliations.
Ready to submit your research? Choose BMC and benefit from:

- fast, convenient online submission

- thorough peer review by experienced researchers in your field

- rapid publication on acceptance

- support for research data, including large and complex data types

- gold Open Access which fosters wider collaboration and increased citations

- maximum visibility for your research: over $100 \mathrm{M}$ website views per year

At $\mathrm{BMC}$, research is always in progress.

Learn more biomedcentral.com/submissions 